\title{
The deformed conifold as a geometry on the space of unit charge $\mathbb{C} P^{1}$ lumps
}

\author{
J.M. Speight \\ Department of Pure Mathematics, University of Leeds \\ Leeds LS2 9JT, England \\ E-mail: j.m.speight@leeds.ac.uk
}

\begin{abstract}
The strong structural similarity between the deformed conifold of Candelas and de la Ossa (a noncompact Calabi-Yau manifold) and the moduli space of unit charge $\mathbb{C} P^{1}$ lumps equipped with its $L^{2}$ metric is pointed out. This allows one to reinterpret certain recent results on D3 branes in terms of lump dynamics, and to deduce certain curvature properties of the deformed conifold.
\end{abstract}

\section{Introduction}

The purpose of this note is to point out that the deformed conifold of Candelas and de la Ossa [2] and the $L^{2}$ geometry on the 1-lump moduli space $\mathfrak{M}_{1}$ of the $\mathbb{C} P^{1}$ model both lie in a certain class of cohomogeneity 1 Kähler geometries on $P L(2, \mathbb{C})$. Consequently, they have strong structural similarities which may be used to transfer results obtained for one to the other, often with a complete change in physical interpretation. For example, their Hodge isomorphisms restricted to cohomogeneous 3 -forms will be shown to coincide. This allows one to transfer a family of harmonic 3-forms constructed by Cvetic et al [1] from the deformed conifold to $\mathfrak{M}_{1}$ changing their interpretation from D3 brane solutions of supergravity to supersymmetric quantum lump states. Thinking of the deformed conifold as a geometry on $\mathfrak{M}_{1}$ also gives an elementary proof that it is the unique scalar flat geometry in its class.

\section{The deformed conifold and $\mathbb{C} P^{1}$ lumps}

The deformed conifold is $T S^{3}$ equipped with its unique $S O(4)$ invariant Ricci-flat Kähler metric, $\widetilde{\gamma}$. Thinking of $T S^{3}$ embedded in $\mathbb{R}^{4} \oplus \mathbb{R}^{4}$ as $\{(X, \xi):|X|=1, X \cdot \xi=0\}$, the isometric $S O(4)$ action is simply

$$
\mathcal{O}:(X, \xi) \mapsto(\mathcal{O} X, \mathcal{O} \xi)
$$


This action has cohomogeneity 1 , the orbits being level sets of $|\xi| \in[0, \infty)$. It may be viewed as a "cone" with link $S^{2} \times S^{3}$, but with the singular tip at $\xi=0$ replaced by $S^{3}$, the single exceptional orbit of the $S O(4)$ action. The complex structure with respect to which $\widetilde{\gamma}$ is Kähler is less obvious; it is obtained by identifying $T S^{3}$ with $S L(2, \mathbb{C})$ via

$$
M:=Z_{0} \mathbb{I}_{2}+i \mathbf{Z} \cdot \boldsymbol{\tau} \in S L(2, \mathbb{C}), \quad Z:=\cosh |\zeta| X+i \frac{\sinh |\zeta|}{|\zeta|} \zeta \in \mathbb{C}^{4}
$$

where $\boldsymbol{\tau}=\left(\tau_{1}, \tau_{2}, \tau_{3}\right)$ are the Pauli spin matrices. Using the isomorphism $\operatorname{Spin}(4) \cong S U(2) \times$ $S U(2)$, one may equally well think of $\widetilde{\gamma}$ as a $S U(2) \times S U(2)$ invariant Kähler metric on $S L(2, \mathbb{C})$, the isometric action here being

$$
(L, R): M \mapsto L M R
$$

Then $\left(-\mathbb{I}_{2}, \mathbb{I}_{2}\right)$ generates a $\mathbb{Z}_{2}$ subgroup acting isometrically, holomorphically and properly discontinuously on $S L(2, \mathbb{C})$ so that $\widetilde{\gamma}$ descends to a $P U(2) \times P U(2)$ invariant Kähler metric $\gamma$ on $P L(2, \mathbb{C})=S L(2, \mathbb{C}) / \mathbb{Z}_{2}$. Conversely $\gamma$ uniquely defines $\widetilde{\gamma}$ by lifting.

Now each $[M]=\{ \pm M\} \in P L(2, \mathbb{C})$ may be uniquely identified with a static $\mathbb{C} P^{1}$ lump on $S^{2}$, that is, a degree 1 holomorphic map $\phi: S^{2} \rightarrow \mathbb{C} P^{1} \cong S^{2}$. Explicitly, one identifies

$$
\pm\left(\begin{array}{ll}
a & b \\
c & d
\end{array}\right) \quad \leftrightarrow \quad W: z \mapsto \frac{a z+b}{c z+d}
$$

where $z, W \in \mathbb{C}$ are stereographic coordinates on the domain and codomain. Map composition and projective matrix multiplication coincide under this identification. Further, the left and right $P U(2)$ multiplication actions on $P L(2, \mathbb{C})$ coincide with the $\mathrm{SO}(3)$ rotation actions on the codomain and domain respectively. Hence $\gamma$ may naturally be interpreted as a $S O(3) \times S O(3)$ invariant Kähler metric on $\mathfrak{M}_{1}$, the 1 lump moduli space. This class of metrics has been studied in depth because $\gamma_{L^{2}}$, the $L^{2}$ metric on $\mathfrak{M}_{1}$ (the restriction to $T \mathfrak{M}_{1}$ of the $\mathbb{C} P^{1}$ model kinetic energy functional), belongs to it [10, 11]. Recall that geodesics in $\left(\mathfrak{M}_{1}, \gamma_{L^{2}}\right)$ are believed to be close to low-energy dynamical solutions of the $\mathbb{C} P^{1}$ model, in analogy with the method of Manton [12, 9].

The most natural coordinate system in which to analyze these metrics is obtained by projecting the standard polar decomposition of $S L(2, \mathbb{C})$ to $P L(2, \mathbb{C})$. That is, each $\{ \pm M\} \in$ $P L(2, \mathbb{C})$ is uniquely decomposed as

$$
M=U\left(\Lambda \mathbb{I}_{2}+\boldsymbol{\lambda} \cdot \boldsymbol{\tau}\right)
$$

where $\pm U \in S U(2), \lambda \in \mathbb{R}^{3}$ and $\Lambda:=\sqrt{1+\lambda^{2}}$. Physically one interprets the map with decomposition $([U], \boldsymbol{\lambda})$ as a lump located at $-\boldsymbol{\lambda} / \lambda \in S^{2}$ with sharpness $\lambda$ and internal orientation $[U] \in P U(2) \cong S O(3)$ [10]. If $\lambda=0$ the map has constant energy density. So these coordinates give a very natural parametrization of $\mathfrak{M}_{1}$ from the physical viewpoint. The $S O(3) \times S O(3)$ action is particularly simple in these terms also:

$$
(\mathcal{L}, \mathcal{R}):([U], \boldsymbol{\lambda}) \mapsto([L U R], \mathcal{R} \boldsymbol{\lambda})
$$

where $[L] \in P U(2)$ is related to $\mathcal{L} \in S O(3)$ by the canonical isomorphism

$$
\mathcal{L}_{a b}=\frac{1}{2} \operatorname{tr}\left(\tau_{a} L^{\dagger} \tau_{b} L\right)
$$


and similarly for $[R]$ and $\mathcal{R}$. One sees once again that the action has cohomogeneity 1 , the orbits being level sets of $\lambda \in[0, \infty)$. Every orbit except $\lambda=0$ is diffeomorphic to $S^{2} \times S O(3)$, while $\lambda=0$ is diffeomorphic to $S O(3)$.

Denoting the usual left-invariant 1 -forms on $P U(2)$ by $\boldsymbol{\sigma}=\left(\sigma_{1}, \sigma_{2}, \sigma_{3}\right)$, the pulled back action on the moving coframe $(\boldsymbol{\sigma}, d \boldsymbol{\lambda})$ is

$$
(\mathcal{L}, \mathcal{R})^{*}:(\boldsymbol{\sigma}, d \boldsymbol{\lambda}) \mapsto(\mathcal{R} \boldsymbol{\sigma}, \mathcal{R} d \boldsymbol{\lambda}) .
$$

So in this coordinate system the problem of constructing $S O(3) \times S O(3)$ invariant $(0, p)$ tensors on $M_{1}$ is reduced to constructing $S O(3)$ scalars of rank $p$ from the 1-form triplets $\boldsymbol{\sigma}, d \boldsymbol{\lambda}$. Applying this idea to any invariant metric, its Kähler form and Ricci tensor, it is possible to prove the following [1]:

Theorem 1 Let $\hat{\gamma}$ be a $S O(3) \times S O(3)$ invariant Kähler metric on $\mathfrak{M}_{1}$ and $\rho$ be its Ricci tensor. Then there exists a smooth function $A:[0, \infty) \rightarrow \mathbb{R}$ such that

$$
\hat{\gamma}=A_{1} d \boldsymbol{\lambda} \cdot d \boldsymbol{\lambda}+A_{2}(\boldsymbol{\lambda} \cdot d \boldsymbol{\lambda})^{2}+A_{3} \boldsymbol{\sigma} \cdot \boldsymbol{\sigma}+A_{4}(\boldsymbol{\lambda} \cdot \boldsymbol{\sigma})^{2}+A_{1} \boldsymbol{\lambda} \cdot(\boldsymbol{\sigma} \times d \boldsymbol{\lambda}),
$$

where $A_{1}, \ldots, A_{4}$ are functions of $\lambda$ only, determined by $A$ as

$$
A_{1}=A(\lambda), \quad A_{2}=\frac{A(\lambda)}{\Lambda^{2}}+\frac{A^{\prime}(\lambda)}{\lambda}, \quad A_{3}=\left(\frac{\Lambda^{2}+\lambda^{2}}{4}\right) A(\lambda), \quad A_{4}=\frac{\Lambda^{2}}{4 \lambda} A^{\prime}(\lambda) .
$$

Further, the Ricci tensor takes the same form, namely,

$$
\rho=\bar{A}_{1} d \boldsymbol{\lambda} \cdot d \boldsymbol{\lambda}+\bar{A}_{2}(\boldsymbol{\lambda} \cdot d \boldsymbol{\lambda})^{2}+\bar{A}_{3} \boldsymbol{\sigma} \cdot \boldsymbol{\sigma}+\bar{A}_{4}(\boldsymbol{\lambda} \cdot \boldsymbol{\sigma})^{2}+\bar{A}_{1} \boldsymbol{\lambda} \cdot(\boldsymbol{\sigma} \times d \boldsymbol{\lambda}),
$$

$\bar{A}_{1}, \ldots, \bar{A}_{4}$ being functions of $\lambda$ only, determined by a single function $\bar{A}$ as in (10), where

$$
\bar{A}=-\frac{2 \lambda \Lambda^{2}\left(A^{\prime}\right)^{2}+\left(9 \lambda^{2}+4\right) A A^{\prime}+\lambda \Lambda^{2} A A^{\prime \prime}+4 \lambda A^{2}}{2 \lambda A\left[\left(\Lambda^{2}+\lambda^{2}\right) A+\lambda \Lambda A^{\prime}\right]} .
$$

In the case of the deformed conifold, $\gamma$ is Ricci flat and hence $\bar{A} \equiv 0$. This is a second order ODE for $A(\lambda)$ which is easily reduced to quadratures. The unique solution which is regular on the exceptional orbit $\lambda=0$ (i.e. with $A^{\prime}(0)=0$ ) is

$$
A(\lambda)=e^{\int_{0}^{\lambda} \beta(\mu) d \mu}, \quad \beta(\lambda)=-\frac{\left(4 \lambda^{5}+4 \lambda^{3}+3 \lambda\right) \Lambda-\left(6 \lambda^{2}+3\right) \sinh ^{-1} \lambda}{3 \lambda\left[\left(2 \lambda^{5}+3 \lambda^{2}+\lambda\right) \Lambda-\left(\lambda^{2}+1\right) \sinh ^{-1} \lambda\right]} .
$$

This should be compared with the $L^{2}$ metric on $\mathfrak{M}_{1}$,

$$
A_{L^{2}}=\frac{\mu\left(\mu^{4}-4 \mu^{2} \log \mu-1\right)}{\left(\mu^{2}-1\right)^{3}}, \quad \mu:=(\Lambda+\lambda)^{2} .
$$

The Ricci flat component function (13) does look somewhat more complicated, but in practice it is often the structure of $\gamma(9)$, (10) which is important rather than the details of $A(\lambda)$. Using the elementary estimate $A(\lambda)>\lambda^{-5 / 6}$ for all $\lambda$ sufficiently large, it is straightforward to verify directly the well-known geometric properties of the deformed conifold (infinite volume and diameter, geodesic completeness). 
Much insight into $\gamma$ may be obtained from $\gamma_{L^{2}}$ and vice versa. We shall see in the next section that a certain family of harmonic forms on $\left(\mathfrak{M}_{1}, \gamma\right)$ transfers directly to $\left(\mathfrak{M}_{1}, \gamma_{L^{2}}\right)$, providing a link bewteen D3 branes and quantum lump dynamics. Other results transfer the other way. For example the explicit formulae (in terms of $A$ ) for holomorphic sectional curvatures, and the analysis of cohomogeneity 1 Hamiltonian flows on $\mathfrak{M}_{1}$ obtained for $\gamma_{L^{2}}$ in [11] apply equally well to the deformed confifold.

\section{Scalar flatness}

One may prove a stronger uniqueness result for the deformed conifold, namely

Theorem 2 The deformed conifold is the unique SO(4) invariant scalar flat Kähler metric on $T S^{3}$.

Since $\kappa=0$ does not imply $\rho=0$ (rather merely $\operatorname{tr} \rho=0$ ) this is a stronger statement than we had previously (unique $S O(4)$ invariant Ricci flat Kähler metric). As shown above, it suffices to prove that $\gamma$ is the unique $S O(3) \times S O(3)$ invariant Kähler solution of $\kappa=0$. From theorem 11 it immediately follows for any invariant Kähler metric on $\mathfrak{M}_{1}$ that

$$
\kappa=4 \frac{\bar{A}}{A}+2 \frac{\bar{B}}{B}
$$

where

$$
B=\frac{1}{4}\left[\left(\Lambda^{2}+\lambda^{2}\right) A+\lambda \Lambda A^{\prime}\right]
$$

and $\bar{B}$ is similarly defined in terms of $\bar{A}[11]$. Substituting (12) and (16) into the scalar flat condition, $\kappa=0$, it reduces to a single third order nonlinear ODE for $A$, to which we seek nonsingular, strictly positive solutions.

Assume there exists a point at which $\bar{A} \neq 0$. Then by continuity, there exists an interval on which $\bar{A} \neq 0$. On this interval, we may rearrange $\kappa=0$, using (15), so that

$$
\begin{aligned}
\frac{\bar{B}}{\bar{A}} & =-2 \frac{B}{A} \\
\Rightarrow \frac{1+2 \lambda^{2}}{4}+\frac{\lambda+\lambda^{3}}{4} \frac{\bar{A}^{\prime}}{\bar{A}} & =-2\left(\frac{1+2 \lambda^{2}}{4}+\frac{\lambda+\lambda^{3}}{4} \frac{A^{\prime}}{A}\right) \\
\Rightarrow \frac{d}{d \lambda}\left[\log \left(A^{2} \bar{A}\right)\right] & =-3 \frac{\left(1+2 \lambda^{2}\right)}{\lambda+\lambda^{3}} \\
\Rightarrow A^{2} \bar{A} & =\frac{C}{(\lambda \Lambda)^{3}}
\end{aligned}
$$

where $C>0$ is a constant. Now $\bar{A}$ cannot vanish at the ends of the interval, or else $A$ is singular, by (17). Hence, the interval must be $(0, \infty)$, that is, if $\bar{A} \neq 0$ anywhere, then $\bar{A}$ vanishes nowhere. But then (17) holds globally, so $A$ or $\bar{A}$ or both must be singular at $\lambda=0$, a contradiction. Hence, all regular solutions of $\kappa=0$ have $\bar{A}=0$ everywhere, and are thus Ricci flat. The metric $\gamma$ constructed above being the unique such metric, the theorem is proved. 


\section{Harmonic 3-forms}

The original motivation behind the construction of $\left(T S^{3}, \widetilde{\gamma}\right)$ was to find a nontrivial CalabiYau manifold to be used as the transverse space to Minkowski space $\left(\mathbb{R}^{4}, \eta\right)$ in superstring theory [2]. It has excited renewed interest recently due to the observation that certain warped products $\left(\mathbb{R}^{4} \times T S^{3}, \alpha \eta \oplus \alpha^{-1} \widetilde{\gamma}\right)$, where $\alpha: T S^{3} \rightarrow \mathbb{R}$, have the interpretation of D3-brane solutions of supergravity [8]. The key step in constructing such a solution is to find a (complex) self dual harmonic 3 -form on $\left(T S^{3}, \widetilde{\gamma}\right)$; this in turn determines $\alpha$. Those forms of pure holomorphicity type are interpreted as supersymmetric solutions.

It turns out that the Hodge isomorphism $*: \bigwedge^{3} \mathfrak{M}_{1} \rightarrow \bigwedge^{3} \mathfrak{M}_{1}$ restricted to $S O(3) \times$ $S O$ (3) invariant 3-forms is independent of $A$. This is somewhat surprising: although the Hodge automorphism on middle rank forms in even dimensions is always conformally invariant, the different metrics introduced in theorem 10 are not necessarily conformally related. In fact, assuming a normalization such as $A(0) \equiv 1$, no two metrics are conformally equivalent. The invariance of $*$ stems from the block structure of $\hat{\gamma}$ and the way in which the space of $S O(3) \times S O(3)$ invariant 3 -forms sits in $\bigwedge^{3} \mathfrak{M}_{1}$. It certainly does not extend to general 3 -forms. In any case, an invariant 3 -form is harmonic with respect to $\gamma$ if and only if it is harmonic with respect to $\gamma_{L^{2}}$. Cvetic et al, as part of a much bigger analysis of Stenzel metrics (Ricci-flat Kähler metrics on $T S^{n}, n=2,3, \ldots$ ), have constructed a 4dimensional family of such forms (although they do not mention $S O(4)$ invariance) which transfers directly to $\left(\mathfrak{M}_{1}, \gamma_{L^{2}}\right)$. How might one interpret them in this new setting?

The standard quantum mechanics of Bogomol'nyi solitons is determined [5] by a covariant Schrödinger equation for a wavefunction $\psi$ on the soliton moduli space, in this context $\psi: \mathfrak{M}_{1} \times \mathbb{R} \rightarrow \mathbb{C}$ where

$$
i \frac{\partial \psi}{\partial t}=-\frac{1}{2} \Delta_{\gamma_{L}} \psi
$$

Gauntlett [4] has proposed, in the case of $\sigma$-models with Kähler target space, a supersymmetric generalization of this in which $\psi$ is an antiholomorphic $p$-form, where $p=$ $0, \ldots, \operatorname{dim}_{\mathbb{C}} \mathfrak{M}_{1}=3$, and $\Delta_{\gamma_{L^{2}}}$ is replaced by the Laplacian on forms $d d^{\dagger}+d^{\dagger} d$ (here $d^{\dagger}:=* d *$ ). Hence antiholomorphic harmonic 3 -forms have an alternative interpretation as supersymmetric quantum lump states which saturate the classical Polyakov energy bound, with the maximal number of fermionic zero modes excited.

The easiest way to translate the relevant results of [1] into $\mathbb{C} P^{1}$ model language is to rederive them, emphasising the $S O(3) \times S O(3)$ invariance property. This will also allow us to strengthen the results slightly: we will show that the 4-dimensional space we construct exhausts the invariant 3 -forms which are closed and co-closed.

Let $\psi$ be a $S O(3) \times S O(3)$ invariant 3 -form on $\mathfrak{M}_{1}$. Then $\psi$ is uniquely determined by specifying its value at one point on each orbit, for example at $M_{\lambda}=\Lambda \mathbb{I}_{2}+\lambda \tau_{3}, \lambda \geq 0$. In other words, $\psi$ is uniquely determined by the 1-parameter family of alternating trilinear forms $\psi_{\lambda}: V_{\lambda} \oplus V_{\lambda} \oplus V_{\lambda} \rightarrow \mathbb{R}$, where $V_{\lambda}:=T_{M_{\lambda}} \mathfrak{M}_{1}$. Further, each $\psi_{\lambda}$ must be invariant under the isotropy group $H_{\lambda}<S O(3) \times S O(3)$ of $M_{\lambda}$, explicitly,

$$
H_{\lambda}= \begin{cases}\left\{\left( \pm \exp \left(-\frac{i}{2} \theta \tau_{3}\right), \pm \exp \left(\frac{i}{2} \theta \tau_{3}\right)\right): \theta \in \mathbb{R}\right\} \cong S O(2) & \lambda>0 \\ \left\{\left( \pm U^{\dagger}, \pm U\right):\{ \pm U\} \in S U(2) / \mathbb{Z}_{2}\right\} \cong S O(3) & \lambda=0\end{cases}
$$


Now the character of the induced representation of $H_{\lambda}$ on $\bigwedge^{3} V_{\lambda}^{*}$ is

$$
\chi(\theta)=8+8 \cos \theta+4 \cos 2 \theta .
$$

Note that this formula holds for all $\lambda$; if $\lambda=0$ it is to be interpreted as a function on $S O(3)$ independent of the axis of rotation $\widehat{\boldsymbol{n}} \in S^{2}$ and depending only on the angle of rotation $\theta$. Taking the character inner product of $\chi$ and the trivial character $\chi_{0}(\theta)=1$ with respect to the Haar measure $d \mu_{\lambda}$ on $H_{\lambda}\left(d \mu_{\lambda}=(2 \pi)^{-1} d \theta, \lambda>0 ; d \mu_{0}=\pi^{-1} \sin ^{2}(\theta / 2) d \theta\right)$ one finds that the subspace of $\bigwedge^{3} V_{\lambda}^{*}$ on which $H_{\lambda}$ acts trivially, call it $W_{\lambda}$, has dimension

$$
\operatorname{dim} W_{\lambda}=\int_{H_{\lambda}} \chi_{0} \chi d \mu_{\lambda}=\left\{\begin{array}{cc}
8 & \lambda>0 \\
4 & \lambda=0
\end{array}\right.
$$

Let $\mu_{1}, \mu_{2}$ denote the invariant 1-forms $\mu_{1}:=\boldsymbol{\lambda} \cdot d \boldsymbol{\lambda}$ and $\mu_{2}:=\boldsymbol{\lambda} \cdot \boldsymbol{\sigma}$, and $\nu$ denote the invariant 2-form $\nu:=d \boldsymbol{\lambda} \cdot \boldsymbol{\sigma}-\boldsymbol{\sigma} \cdot d \boldsymbol{\lambda}$. Consider the following eight 3-forms:

$$
\begin{gathered}
\rho_{1}^{-}=d \lambda_{1} \wedge d \lambda_{2} \wedge d \lambda_{3}, \quad \rho_{2}^{-}=\mu_{1} \wedge \nu, \quad \rho_{3}^{-}=d \boldsymbol{\lambda} \cdot(\boldsymbol{\sigma} \times \boldsymbol{\sigma}), \quad \rho_{4}^{-}=\mu_{1} \wedge \boldsymbol{\lambda} \cdot(\boldsymbol{\sigma} \times \boldsymbol{\sigma}), \\
\rho_{1}^{+}=\boldsymbol{\sigma} \cdot(d \boldsymbol{\lambda} \times d \boldsymbol{\lambda}), \quad \rho_{2}^{+}=\mu_{1} \wedge \boldsymbol{\lambda} \cdot(d \boldsymbol{\lambda} \times \boldsymbol{\sigma}+\boldsymbol{\sigma} \times d \boldsymbol{\lambda}), \quad \rho_{3}^{+}=\mu_{2} \wedge \nu, \quad \rho_{4}^{+}=\sigma_{1} \wedge \sigma_{2} \wedge \sigma_{3} .
\end{gathered}
$$

The superscripts \pm denote the decomposition of $\bigwedge^{3} \mathfrak{M}_{1}$ into \pm 1 eigenspaces of the pullback $\mathrm{P}^{*}$ of the antiholomorphic isometric involution $\mathrm{P}: M \mapsto \bar{M}$ (entrywise complex conjugation). Clearly $\rho_{a}^{ \pm}$are by construction $S O(3) \times S O(3)$ invariant. Furthermore, their restrictions $\left.\rho_{a}^{ \pm}\right|_{M_{\lambda}}$ span $W_{\lambda}$ for all $\lambda \geq 0$ by (21). Hence any invariant 3 -form $\psi$ may be decomposed as

$$
\psi=\sum_{a=1}^{4}\left(C_{a}^{-} \rho_{a}^{-}+C_{a}^{+} \rho_{a}^{+}\right)
$$

where $C_{a}^{ \pm}$are functions of $\lambda$ only.

We now construct the Hodge isomorphism restricted to such invariant forms. It suffices to construct $*: W_{\lambda} \rightarrow W_{\lambda}$. Note that $\mathrm{P}^{*}$ vol $=-$ vol (vol being the volume form) and by definition

$$
* \psi \wedge \omega=\langle\psi, \omega\rangle_{\gamma} \text { vol }
$$

for all $\omega$, so $*$ must have the block structure

$$
*=\left[\begin{array}{cc}
0 & *_{+} \\
*_{-} & 0
\end{array}\right]
$$

where $*_{ \pm}: W_{\lambda}^{ \pm} \rightarrow W_{\lambda}^{\mp}$ and $W_{\lambda}=W_{\lambda}^{-} \oplus W_{\lambda}^{+}$again denotes the eigenspace decomposition with respect to $\mathrm{P}^{*}$. Straightforward linear algebra establishes that

$$
\begin{aligned}
& *_{+}=\frac{1}{\Lambda^{3}}\left[\begin{array}{cccc}
-2 \lambda^{2} & 0 & 8 \lambda^{2} & -8 \\
-\left(\Lambda^{2}+\lambda^{2}\right) & 0 & 2 \Lambda^{2}+4 \lambda^{2} & -4 \\
-\Lambda^{4} / 2 & -\lambda^{2} \Lambda^{4} / 2 & 0 & 0 \\
-\left(\Lambda^{2}+\lambda^{2} / 2\right) & \Lambda^{4} / 2 & 2\left(\Lambda^{2}+\lambda^{2}\right) & -2
\end{array}\right], \\
& *_{-}=\frac{1}{\Lambda}\left[\begin{array}{cccc}
\lambda^{2} / 2 & -2 \lambda^{2} & 2 & 2 \lambda^{2} \\
-1 / 2 & 2 & 0 & -2 \\
\left(\Lambda^{2}+\lambda^{2}\right) / 4 & \left(\Lambda^{2}+2 \lambda^{2}\right) / 2 & 1 & \lambda^{2} \\
\left(\Lambda^{2}+\lambda^{2}\right)^{2} / 8 & -\lambda^{2}\left(\Lambda^{2}+\lambda^{2}\right) / 2 & \lambda^{2} / 2 & \lambda^{4} / 2
\end{array}\right]
\end{aligned}
$$


relative to the bases (22). Note that, as claimed above, $*$ is independent of $A$, and that $*_{-} *_{+}=*_{+} *_{-}=-\mathbb{I}_{4}$ so that $* *=-\mathrm{Id}$, as of course it must.

To obtain a harmonic 3 -form $\psi$, it is sufficient (but not necessary, $\mathfrak{M}_{1}$ being noncompact) to demand that $\psi$ be closed and co-closed $(d \psi=d * \psi=0)$. Using the notation of (23),

$$
\begin{aligned}
d \psi= & \left(C_{2}^{-}+\frac{\dot{C}_{3}^{-}}{\lambda}-C_{4}^{-}\right) \mu_{1} \wedge \rho_{3}^{-}+\left(\frac{\dot{C}_{1}^{+}}{\lambda}-2 C_{2}^{+}\right) \mu_{1} \wedge \rho_{1}^{+}+\left(\frac{\dot{C}_{3}^{+}}{\lambda}-C_{2}^{+}\right) \mu_{1} \wedge \rho_{3}^{+} \\
& +\frac{\dot{C}_{4}^{+}}{\lambda} \mu_{1} \wedge \rho_{4}^{+}+\left(C_{3}^{+}-\frac{C_{1}^{+}}{2}\right) \nu \wedge \nu
\end{aligned}
$$

where $\dot{C}_{a}^{ \pm}:=d C_{a}^{ \pm} / d \lambda$. So $\psi$ is closed if and only if

$$
\begin{aligned}
& C_{2}^{-}+\frac{\dot{C}_{3}^{-}}{\lambda}-C_{4}^{-}=0 \\
& C_{1}^{+}=2 C_{3}^{+}, \quad \dot{C}_{3}^{+}=\lambda C_{2}^{+}, \quad \dot{C}_{4}^{+}=0 .
\end{aligned}
$$

Clearly $\psi$ is closed and co-closed if and only if $* \psi$ is closed and co-closed. Furthermore, by the block structure of $*$, if $\mathrm{P}^{*} \psi=\psi$ then $\mathrm{P}^{*} * \psi=-* \psi$, so it suffices to find all closed and co-closed $\mathrm{P}^{*}$-even 3 -forms, $\psi^{+}=\sum C_{a}^{+} \rho_{a}^{+}$. Applying (29) to the coefficients of $\psi^{+}$, then (28) to the coefficients of $* \psi^{+}$, one obtains a system of ODEs for $C_{a}^{+}$, the most general solution to which, assuming nonsingularity on the exceptional orbit $\lambda=0$, is

$$
\psi^{+}=a t_{1}+b t_{2}
$$

where $a, b \in \mathbb{R}$ are constants, and

$$
\begin{aligned}
& t_{1}=y(\lambda) \rho_{1}^{+}+\frac{y^{\prime}(\lambda)}{2 \lambda} \rho_{2}^{+}+\frac{1}{2} y(\lambda) \rho_{3}^{+}, \quad y(\lambda)=2+\frac{1}{\lambda^{2}}\left[1-\frac{\log (\lambda+\Lambda)}{\lambda \Lambda}\right] \\
& t_{2}=-4 \rho_{1}^{+}-2 \rho_{3}^{+}+\rho_{4}^{+} .
\end{aligned}
$$

The most general $\mathrm{P}^{*}$-odd closed, co-closed invariant 3-form is then

$$
\psi^{-}=a^{\prime} * t_{1}+b^{\prime} * t_{2} .
$$

So the whole family is 4-dimensional, and presumably coincides with that constructed in [1]. Given any form in the complexification of this family (i.e. allowing $a, b, a^{\prime}, b^{\prime} \in \mathbb{C}$ ) one may construct a complex (anti-) self dual harmonic form

$$
\widetilde{\psi}=\psi \mp i * \psi, \quad * \widetilde{\psi}= \pm i \widetilde{\psi}
$$

as required for a D3 brane.

We reiterate that these forms are harmonic with respect to any $S O(3) \times S O(3)$ invariant Kähler metric on $\mathfrak{M}_{1}$, including $\gamma_{L^{2}}$. Of particular interest in this context, as explained above, are antiholomorphic forms. Since $\mathfrak{M}_{1}$ supports a Calabi-Yau metric, the canonical bundle $\bigwedge^{(3,0)} \mathfrak{M}_{1}$ is trivial. Let us introduce the invariant holomorphic 1-forms

$$
\epsilon_{a}:=\sigma_{a}-i J^{*} \sigma_{a}
$$


where $J^{*}$ is the pullback of the almost complex structure $J: T_{M} \mathfrak{M}_{1} \rightarrow T_{M} \mathfrak{M}_{1}$ as found in [11] (in matrix terms, $J^{*}=J^{T}$ ). Explicitly, one sees that at $M_{\lambda}$,

$$
\epsilon_{1}=\sigma_{1}-\frac{i}{\Lambda}\left(2 d \lambda_{1}-\lambda \sigma_{2}\right), \quad \epsilon_{1}=\sigma_{2}-\frac{i}{\Lambda}\left(2 d \lambda_{2}+\lambda \sigma_{1}\right), \quad \epsilon_{3}=\sigma_{3}-\frac{2 i}{\Lambda} d \lambda_{3},
$$

for example. The canonical bundle has nonvanishing global section $\epsilon_{1} \wedge \epsilon_{2} \wedge \epsilon_{3}$. Comparing this at $M_{\lambda}$ with $\left.t_{2}\right|_{M_{\lambda}}$ and appealing to $S O(3) \times S O(3)$ invariance shows that

$$
\epsilon_{1} \wedge \epsilon_{2} \wedge \epsilon_{3}=\frac{1}{\Lambda^{2}}\left(t_{2}-i * t_{2}\right) .
$$

So the anti-self dual 3 form $t_{2}+i * t_{2}$ is harmonic and antiholomorphic. As remarked in [1], $\psi$ is not $L^{2}$ normalizable with respect to the Ricci-flat metric $\gamma$. Since $*$ is independent of $A$ for invariant 3 -forms, so is

$$
\|\psi\|^{2}=\int_{\mathfrak{M}_{1}} * \psi \wedge \psi
$$

and it follows that $\psi$ is not $L^{2}$ normalizable with respect to $\gamma_{L^{2}}$ either. This makes its interpretation as a quantum state problematic (as it does the D3 brane interpretation also). Does it make sense for a non-normalizable state to be BPS? Can one still interpret $\mathcal{P}(\lambda)$, where $* \psi \wedge \psi=\mathcal{P}(\lambda)$ vol, as a probability density?

If not then we have actually proved that there is no invariant BPS 3-form state, i.e. none with maximally excited fermionic zero modes. To see this note that such a state would have to be $L^{2}$ finite, invariant and harmonic, which implies, by self adjointness of $d^{\dagger} d+d d^{\dagger}$ on $L^{2}$, that the state is closed and co-closed, irrespective of the noncompactness of $\mathfrak{M}_{1}$. But we have classified all such forms, and none is $L^{2}$ finite. It would be interesting to perform a similar analysis for invariant $0-, 1$ - and 2-forms. Here the results will depend on $A$, but seem to do so in quite a simple way so that again, translation between different $S O(3) \times S O(3)$ invariant Kähler geometries should be quite straightforward.

\section{Special Lagrangian submanifolds}

The moduli space picture can give considerable geometric insight. For example, consider those holomorphic maps $\phi: S^{2} \rightarrow S^{2}$ which factor through the quotients in

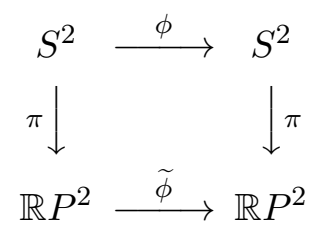

where $\pi$ denotes the covering projection. The projected maps $\widetilde{\phi}$ so generated are precisely the harmonic maps $\mathbb{R} P^{2} \rightarrow \mathbb{R} P^{2}$ [3], or in physics language, static $\mathbb{R} P^{2}$ lumps on $\mathbb{R} P^{2}$. Now $\phi \in \mathfrak{M}_{n}=\operatorname{Hol}_{n}\left(S^{2}, S^{2}\right)$ factors if and only if it is a fixed point of $\Pi: \mathfrak{M}_{n} \rightarrow \mathfrak{M}_{n}$,

$$
\Pi: \phi \mapsto p \circ \phi \circ p
$$

where $p: S^{2} \rightarrow S^{2}$ is the antipodal map. In fact $\Pi$ is an antiholomorphic isometry of any invariant Kähler metric $\hat{\gamma}$, and the fixed point set of $\Pi$, call it $\widetilde{\mathfrak{M}}_{n}$, has exactly half the 
(real) dimension of $\mathfrak{M}_{n}$. Since $\Pi$ is an isometry, $\widetilde{\mathfrak{M}}_{n} \subset \mathfrak{M}_{n}$ is totally geodesic, and hence minimal. Further, since $\Pi$ is antiholomorphic, the restriction of the Kähler form $\Omega=\hat{\gamma}(J \cdot, \cdot)$ to $\widetilde{\mathfrak{M}}_{n}$ vanishes identically on $T \widetilde{\mathfrak{M}}_{n}$ 11]. So $\widetilde{\mathfrak{M}}_{n}$ is a minimal Lagrangian submanifold of $\mathfrak{M}_{n}$. In the case of $\mathfrak{M}_{1}, \widetilde{\mathfrak{M}}_{1}$ is precisely the exceptional orbit $\lambda=0$ (diffeomorphic to $S O(3)$ ), the "bolt" in the language of [1]. Choosing $\hat{\gamma}=\gamma$, the Ricci-flat metric, one has a connected, embedded, minimal Lagrangian submanifold of a Calabi-Yau manifold, which must therefore be special Lagrangian, by a straightforward extension [7] of a theorem of Harvey and Lawson [6] concerning such submanifolds in $\mathbb{C}^{n}$. In this picture, the special Lagrangian bolt is viewed as the space of (twisted degree 1 ) harmonic maps $\mathbb{R} P^{2} \rightarrow \mathbb{R} P^{2}$ sitting inside the space of (degree 1) harmonic maps $S^{2} \rightarrow S^{2}$.

Cvetic et al arrive at the same conclusion (the bolt is special Lagrangian) but via explicit consideration of the calibrated geometry of the holomorphic harmonic 3 -form $t_{2}-i * t_{2}$. The argument above gives an alternative derivation, from a somewhat different geometric viewpoint. Note that its key ingredient is the antiholomorphic isometry $\Pi$ whose fixed point set is 3-dimensional. Another such isometry is the reflexion $\mathrm{P}$ already defined, $\mathrm{P}: M \mapsto \bar{M}$. Its fixed point set, diffeomorphic to $S^{1} \times \mathbb{C}$ [10], is another special Lagrangian submanifold of $\left(\mathfrak{M}_{1}, \gamma\right)$. In terms of the canonical open embedding $\mathfrak{M}_{1} \subset \mathbb{C} P^{3}$, it is simply the intersection of $\mathfrak{M}_{1}$ with the obvious totally real subspace $\mathbb{R} P^{3} \subset \mathbb{C} P^{3}$.

\section{Acknowledgments}

The author wishes to thank Mark Haskins for useful discussions. He is an EPSRC Postdoctoral Research Fellow in Mathematics.

\section{References}

[1] M. Cvetic, G.W. Gibbons, H. Lu and C.N. Pope, "Ricci-flat metrics, harmonic forms and brane resolutions" preprint hep-th/0012011 (2000).

[2] P. Candelas and X.C. de la Ossa, "Comments on conifolds" Nucl. Phys. B342 (1990) 246.

[3] J. Eells and L. Lemaire, "On the Construction of Harmonic and Holomorphic Maps Between Surfaces" Math. Ann. 252 (1980) 27.

[4] J.P. Gauntlett, "Low-energy dynamics of supersymmetric solitons" Nucl. Phys. B400 (1993) 103.

[5] G.W. Gibbons and N.S. Manton, "Classical and quantum dynamics of BPS monopoles" Nucl. Phys. B274 (1986) 183.

[6] R. Harvey and H.B. Lawson, "Calibrated geometries" Acta Math. 148 (1982) 47.

[7] M. Haskins, private communication (2001).

[8] I.R. Klebanov and M.J. Strassler, "Supergravity and a confining gauge theory: duality cascades and $\chi S B$-resolution of naked singularities" JHEP 0008 (2000) 052. 
[9] N.S. Manton, "A remark on the scattering of BPS monopoles" Phys. Lett. 110B (1982) 54 .

[10] J.M. Speight, "Low energy dynamics of a $\mathbb{C} P^{1}$ lump on the sphere" J. Math. Phys. 36 (1995) 796.

[11] J.M. Speight, "The $L^{2}$ geometry of spaces of harmonic maps $S^{2} \rightarrow S^{2}$ and $\mathbb{R} P^{2} \rightarrow$ $\mathbb{R} P^{2}$ " preprint math.DG/0102038 (2001).

[12] R.S. Ward, "Slowly moving lumps in the $\mathbb{C} P^{1}$ model in $(2+1)$ dimensions" Phys. Lett. 158B (1985) 424. 